\title{
Review of Different Techniques used for Wireless Transmission of Electrical Energy
}

\author{
Muhammad Bilal Sarwar ${ }^{1,}$, Perumal Nallagownden ${ }^{1}$, Zuhairi Baharudin ${ }^{1}$ and Mohana Sundaram Muthuvalu ${ }^{1}$ \\ ${ }^{1}$ Department of Electrical and Electronic Engineering, Universiti Teknologi PETRONAS, Malaysia
}

\begin{abstract}
The wireless transfer of electricity has gained vast importance in recent years. Many attempts had been done in this regard. The information generated is quite scattered and distributed. There is a need to harmonize the research findings of various researchers in an adept way. This review paper presents the critical evaluation of the research works done in the field of wireless power transmission since 1891. The attempt has been done in a cohesive way to connect the various findings and discussing their capabilities and limitations. The paper also highlights the major challenges faced by the research area and the new area of applications. The study closes with a conclusive review of latest scientific techniques and theoretical concepts, currently prevalent in wireless power transmission.
\end{abstract}

\section{Introduction}

Wireless electricity or Witricity, as expressed in its condensed form, is a developed technology for transmission of electrical power in wireless environment, having its roots in the past. The innovation can be credited to Nikola Tesla who invented a technique for the transmission of power in absence of wires in 1891 [1]. The system operates in conjunction with the principle of radio receivers and that the device must be placed within the range of the transmitter. Such transfer of power is classified as Non-radioactive wireless power transfer. This relies on the near field magnetic coupling of conductive loops. It has sub-types defined as short range and midrange applications. The principle of resonant objects resonating energy efficiently while non-resonant objects remain adamant forms the basic working principle for such systems.

The application wireless transfer in $\mathrm{AC}$ machines has been abundantly reported by Nikola Tesla [1]. For instance, the energy transfer takes place from the excited stator to rotor cage (through their air gaps) in a cage induction machine. Electric machines are thus based upon the principle of Energy transfer via coupled windings. A Very simple circuit diagram is given in Fig. 1 that shows the complete phenomena behind this theory.

Primary and secondary coil constitutes the basic system. Primary coil act as a transmitter and secondary coil is a receiver or load. Tesla's idea of a capacitance loaded open secondary circuit is highlighted in the mid of 20th century [2]. It is of greater criticality that advantageous presence of intra-winding capacitance of the receiver coil for magnetic resonance in the time of Tesla would have helped him to work with several tens of Mega Hertz frequency.

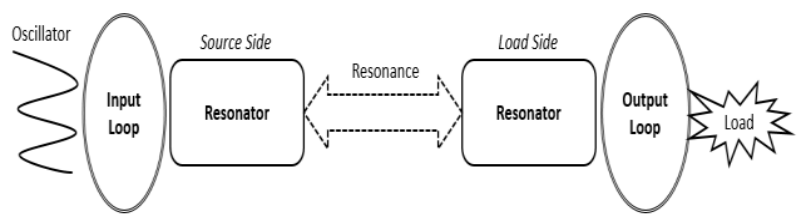

Figure 1. Schematic Circuit Diagram of Tesla's Wireless Power Experiments

The interest for wireless technology has grown in recent years. Specifically in a field of inductively coupled systems we observed significant progress has been done. The development of inductively coupled power transfer systems for a wide range of applications has led to a very high end to end system efficiency of up to $80 \%$. Such examples include battery charging for automobile systems. The limitation is however the restriction of close ranges which is typically believed to be less than $30 \%$ of the coil diameter. The destined distance for transmission is kept below $1 \mathrm{~cm}$ in most cases while $15 \mathrm{~cm}$ is treated as fairly lager distance [4].

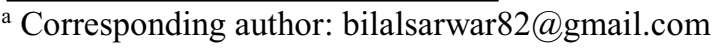


The launch of "Qi" standard by the Wireless Power Consortium has helped in the commercialization of charging technology for portable devices [3]. In this review paper, research on wireless power transmission is briefly summarized and then different techniques applied on this research are discussed. In the later part of this paper several observations are presented regarding practical issues that are deemed necessary for implementation of engineering. The paper closes with an attempt to link the theory and practice together.

\section{Capabilities and limitations of research works a chronological review}

The wireless transfer of power has been recently exploited field of research which has received immense importance with the turn of the century. The work in the field can be traced back to discoveries and patented inventions done by Nikola Tesla [2] in year 1891.

The pioneering work of Tesla is regarded as the focal point for the base case development of "oscillation transformer". The work was oriented to couple both receiver and transmitter in such a resonant mode that they could receive and transmit signals efficiently. Although the scope of work was limited to medium range radio frequency (Tesla self-designated his use of frequency as high frequency), the result outcomes paved a way for future research and a fortiori is found between all the future works that this work surely formed the founding principles for both non-radioactive and radioactive wireless transfer, even though Tesla self-acknowledged his research limitations which was the inability to transfer power beyond/over obstacles.

The spectrum of Tesla's research deviated from power transfer to a development of a field of research that we recognize as signal transmission. The use of low power over large distances was researched in detail, leading to transfer of power as modulators or source of information. On the other hand, the research in power transmission lagged due to focal research being carried out in development of better electrical conductors for wires. The topic again gained importance with the failure of development of super conductors at ambient temperature and pressure conditions in late nineties and even if successful, the associated cost was impractical. Meanwhile, small scale power transmission applications progressed.

It is a well-known fact that wireless power transmission developed more as an application rather than a scientific theory. This can be seen through a number of research applications in biomedical [2-6], transport and signals from 1917 to 1960 's and even later. A milestone research work was presented by William C. Brown in 1961. The paper presented a methodical way of transmitting power at microwave level. The probable selection of such a small frequency can be attributed to inherent advantage of larger distances being covered. On the other hand, the method was limited by transmission of low level power and with decreased efficiency. Some of the other problems faced by this time were the associated misalignment of power transmitter and receiver devices, which further generated an uncertainty in power transfer reducing the efficiency.

The breakthrough research work of Hochmair [4] resolved the aforementioned limitations. Although his work was more relevant to biomedical application of power transmission, he explained the effect of various geometries in resolving the misalignment of frequencies in transmitter and receiver devices.

The work of Green and Boys [5] and his colleagues in the field of power transfer in transport systems is another landmark for wireless transmission. The work mostly attenuated the concept and control parameters associated with power transmission, but were limited to medium power and short distances. The cost associated with the system was also high and the scenarios of work mode were mostly limited to small scale low power applications.

In 2008, the revolutionary work of Zhu et al. [6] paved a new technology innovation in the field of wireless power transmission. The researchers utilized the concept of back emf in the receiving coil. This innovation helped in increasing the transfer distance and the transmitted power, even at medium radio frequency range. One of the greatest breakthroughs of this technique was the advantage of power being transferred over/beyond obstacles. Although, such milestone has already been achieved in signals transmission but the wireless power transmission still lagged in it. The resultant limitation of using back emf was the creation of eddy currents in the transmitting magnetic field due to presence of metallic objects. This severely hammered the performance of the systems in urban or developed sites.

The use of resonant coupling technology gained importance in wireless power transmission with the work of Kim et al. [7]. He utilized the concepts of Nikola Tesla to develop highly efficient wireless power transmission systems. Interestingly, he showed to transmit power over distances of 2 meters with low efficiency of $20 \%$. Although this efficiency was low, the transmitted power ranged in nominal value of $50 \mathrm{~W}$ with a high range radio frequency of $9.4 \mathrm{MHz}$. The work was limited by increased effect of radiation and the difficulty associated with fabrication.

The work of Fu et al. [8] in the same year showed a similar performance. Although the distances covered were not so great $(5 \mathrm{~cm})$ with a mere efficiency of $35 \%$, the better outcome of his research was the study on the effect of coil radius, coil thickness and coil turns on the transfer efficiency.

The work of Cannon et al. [9] exploited the potential of wireless power transmission from a single point source to multiple receivers. This helped in the understanding of the distribution of power among various simultaneous receivers and receivers placed at various distances. The work also provoked the idea of multiple charging and multiple systems based upon single power transmitting source. The system was rather weak, poorly developed and had various design flaws. With the end of year 2009, various conceptual aspect and theoretical insights were 
understood about wireless power transmission that actually developed from applications. The improvement in the experimental findings of $\mathrm{Kim}$ et al. [7] was proposed by the research work of Beh et al. [10]. The researchers used impedance matching technology to increase the efficiency of wireless power transmission systems, via magnetic resonance methodology. For this purpose, high frequency was used in order to avoid internal losses. The reason of the selection of this high frequency system was due to the limitation of electromagnetic induction for short ranges and low efficiency observed for microwave based wireless energy transfer.

Beh furthered his work on wireless power transmission in the same year by implementing fixed resonance frequency at $13.56 \mathrm{MHz}$ [17]. It helped in improving the power transfer by improving the efficiency up to $40 \%$ by this technique, over the same distances. This was achieved by using impedance matching circuit for various air gaps and distances.

Up till this time, the attempts in description of wireless power transmission systems were scattered and the information was highly distributed. In 2011, the work of Ho et al. brought harmony to the distribution of work and presented a comparative study of the two prevalent methodologies, currently utilized [11]. The authors compared the functionality and physical attributes of novel witricity and traditional inductive magnetic coupling and presented their capabilities and drawbacks. The work can be regarded cohesive but connection within the techniques and history was poor. A similar attempt was done by Burali and Patil [12].

The technology behind magnetic resonance coupling lies in magnetic field propagation between a transmitter and a receiver at the same frequency called resonant frequency. The principal is focused on resonating the energy back and forth in a specified area of effect in which enables a strongly coupled magnetic field, transferring energy at the highest efficiency. The rest follows Faraday's Law and Lenz's Law for electromagnetic induction $[13,14,15]$

Based on [16], the frequency of resonance will affect the Q-factor of the resonator that is also influenced by inductance and resistance of coil. With higher Q-Factor, the power transfer efficiency will increase due to higher coupling effect [16]. Following that, the inductance and capacitor used to obtain resonance effect will produce LC cancellation that causes the resistance across the $\mathrm{L}$ and $\mathrm{C}$ to be low at resonant frequency and this increases Qfactor [17]. According to [16] and [17], the power efficiency is affected by design parameters of transmitter and receiver coil focusing on increasing Q-Factor and resonant frequency configuration.

Apart from resonant frequency selection and coil design, the waveform highlighted by [13], [14], [18] and [15] for magnetic field induction in accordance to Faraday's Law is a sine wave. In fact, researched done by [15] have discovered that sine wave provided highest efficiency compared to other waveforms. The sine wave input to the transmitter coil in radiating magnetic field was accomplished by using an oscillator with adjustable duty cycle to convert DC source into alternating current of significant power and frequency [13], [18]. According to [14], the wireless power transmission efficiency was inversely proportional to the duty cycle after the maximum duty cycle have been achieved. Furthermore, [13] have mentioned a $15 \%$ estimated efficiency of implemented oscillator in which [18] have resorted to using power amplifier to increase efficiency.

Despite the success of generating magnetic field, performance of WPT system as a whole is questioned by [17] with consideration on coil performance but also regarding power efficiency of power electronics circuit that generated the sine wave. [17] emphasized the need to consider power losses before the coil on the basis that a decrease in output power of circuit components will be limiting power flowing to the coil. Furthermore, based on magnetic field effect, the magnetic field strength is directly proportional to current [19] and with high power losses in circuit components, the coil will be hindered from maximum magnetic field generation in which justifies concerns of [17] in the importance of considering power electronics efficiency.

In designing a high efficiency coil, [17] and [16] have emphasized the requirement to have high Q-Factor by increasing inductance and reducing resistance value. The multilayer coil proposed by [20], [21] and [22] consists of stacked layers of coils on top of each other where the cross sectional area increases with each stack. Following that, the internal resistance decreases as the stack of coil increases [21]. Moreover, the inductance will increase while diameter of coil remain unchanged, contributing to high efficiency small coil design in which is suitable for WPT operation [23].

Despite the high efficiency and low internal resistance of multilayer coil, the design requires consideration in terms of skin effect, proximity effect and displacement current $[22,23]$. Although a higher frequency increases Q-Factor $[17,16]$, the increase in frequency of operation will increase AC resistance due to skin effect and proximity effect that in fact decreases Q-Factor [22, 23]. Furthermore, [23] have discovered the increase in AC resistance is most distinct when the stacked layers are increased beyond the maximum amount.

Based on the fact that there is a maximum stacked layer in which varies for different coil dimensions, [20] and [24] demonstrates brook's coil design that is a multilayer coil orientation with a square cross sectional area and controlled dimensions by ratio, standardized the amount of stacked layer for maximum performance. This type of design enables optimum inductance value at a finite length of wire [20].

In spite of brook's coil and multilayer coil design, the use of magnetic core for WPT coil design was not effective because of core saturation [20] and negative effect to surrounding wireless devices [22]. With that, [20] and [22] have justified using air core in which is efficient and does not affect inductance with an increase in current. Therefore, consideration is required in terms of frequency of operation with number of stack layers and the application of core in coil design is crucial. 


\subsection{Power Electronics Circuit - Power Amplifier}

Power amplifiers are categorised into linear power amplifiers such as class $\mathrm{A}, \mathrm{B}$ and $\mathrm{AB}$ and switching amplifiers with class $\mathrm{C}, \mathrm{D}, \mathrm{E}$ and $\mathrm{F}$. In the case of WPT application, the switching amplifier produces substantial improvement towards power efficiency especially related to class E power amplifier design. The design process is tedious although simple to construct with success in WPT implementation [25-26].

Basically, the class E power amplifier uses lesser components compared to other classes in the same category and achieve higher efficiency [25]. According to [26], the calculation in design consists of long iterative methods in which is a setback to simple circuit construction. In order to fully comprehend the advantages of class E power amplifiers, a simple analytical design method was implemented by [26] to produce a high approximation design. However, the full and complete mathematical model is covered by [25].

With the design process made simple, there have been success in implementing this power amplifier in WPT system. [27] and [28] have significantly improved WPT system efficiency to above $80 \%$ by using class E power amplifier. The improvement of system efficiency was due to reduced power dissipation in power amplifier through zero voltage switching [25] that increased power transfer to coils [27-28].

Therefore, concerns in power efficiency of circuit components by [26] was addressed by class E power amplifier application in WPT. However, the application on WPT using magnetic resonance coupling at low frequency range have not been explored and due to frequency effect on coil resistance, the research on this will be useful in developing high efficiency WPT system at low frequency range.

\section{Methods used for Witricity}

We have major four techniques used for transmission of electric energy wirelessly which are given below.

1. Wireless power systems with 2 coil-resonators.

2. Wireless power systems with 2 coil resonators and input and output impedance matching.

3. Wireless power systems with relay resonators.

4. Wireless power domino-resonator systems.

In all the above mention methods we have two objects that should be achieved that are transferring the maximum Power and Efficiency to our receiving or load side. Maximum power transfer theorem regulates the calculation of maximum power. Fig. 2 shows the equivalent circuit of an electric system. Source and load impedance are $R S+j X S$ and $R L+j X L$ respectively [14].

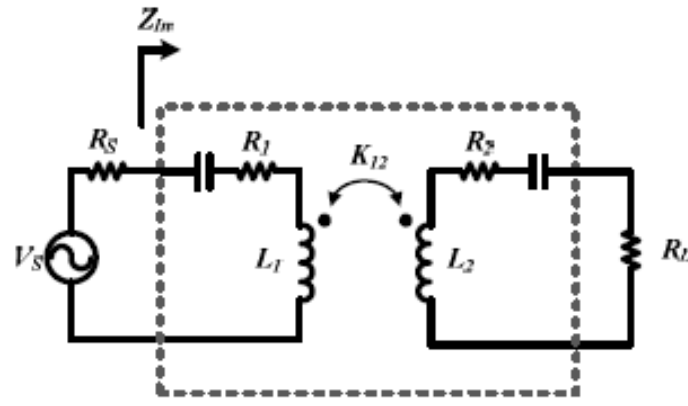

Figure 2. Equivalent Circuit of an Electrical System

Then maximum power can be delivered to the load if $\mathrm{RS}=\mathrm{RL}$ and $\mathrm{XS}=\mathrm{XL}$. This is the most selected approach by the recent research works in the echelon of mid-range wireless power transfer. However, the equation (1) limits the application of such approach due to its incapacity to represent maximum energy efficiency not greater than $50 \%$ [1]. For RS=RL,

$$
\eta=\frac{i^{2} R_{L}}{i^{2} R_{S}+i^{2} R_{L}}=\frac{R_{L}}{R_{S}+R_{L}}=0.5
$$

\section{Some other Techniques Relate with Witricity}

\subsection{Electricity}

This phenomenon arises from the behaviour of electrons and protons that are used the attraction of particles with opposite charges and the repulsion of particles with the same charges [29].

\subsection{Magnetism}

Magnetism is a phenomenon which explains the attraction or repulsion of materials and the fundamental force of nature associated. The suitable examples of permanent magnets (that exhibit constant magnetic fields) may include earth's magnetic field or the one in your refrigerator. The alternating current is known to generate oscillating magnetic fields, which varies with period division and time.

\subsection{Electromagnetism}

A term for the interdependence of time-varying electric and magnetic fields for example, it turns out that an oscillating magnetic field produces an electric field and an oscillating electric field produces a magnetic field. With flow of electric current in a wire, magnetic field is generated. This field wraps around the wire. It is a wellknown fact that as the reversal in direction of current reverses the magnetic field [1]. 


\subsection{Magnetic Induction}

It is defined as the quantitative value of magnetic flux in a unit area perpendicular to the direction of magnetic flow [29]. Electric transformers and generators follow the same principle.

\subsection{Power Coupling}

Energy coupling or power coupling is a transfer of electrical energy from one circuit segment to another circuit [13]. The phenomenon of interaction of magnetic field of two objects and consequent induction of electric current on either of the object is known as magnetic coupling. Thus, using this phenomenon, energy transfer can be initiated from a power source to a power device. Thus, it does not require physical contact between both objects.

\subsection{Resonant Magnetic Coupling}

In case, the natural frequencies of the two magnetic field generating objects coincide, the resultant magnetic coupling is known as resonant magnetic coupling.

\subsection{Magnetic Field Repeater}

In WPT system, the higher the magnetic field received by receiver, the higher the efficiency. However, the long separation distance between transmitter and receiver causes the low magnetic coupling effect which results short transmission distance of WPT system. This is because insufficient magnetic field is captured by receiver which causes it unable to power up the load. One of the alternatives to overcome this issue is by inserting a relay resonator in between the transmitter and receiver $[8$, $15,16]$. The relay resonator can enhance the magnetic coupling by concentrating and relaying the magnetic flux to a longer distance. As shown in fig. 3 and also stated in [8], it shows that for the odd number of repeater, the system achieve its optimum performance at the original resonant frequency but it does not apply on even number of repeaters. Total number of repeaters has to be carefully selected to avoid frequency splitting effect which will reduce the overall transmission efficiency.

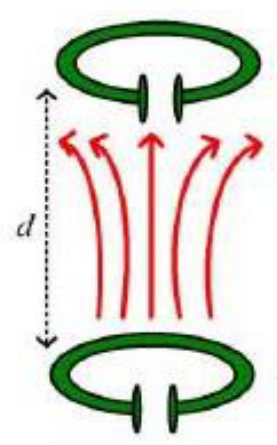

Device Coil

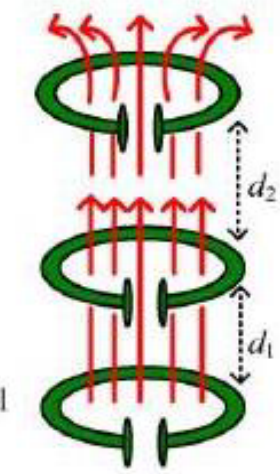

Figure 3. Magnetic Field Repeater

\section{System Overview of Witricity}

It is acknowledged widely that WiTricity is actually wireless electricity. The probable applications of WiTricity may include the cell phones, laptops, iPods and other power hungry devices. This will make them to get charged on their own, eliminating the requirement of plugging them for charging [30,31,32]. The whole phenomena of Witicirty is shown in the form of block diagram in fig. 4.

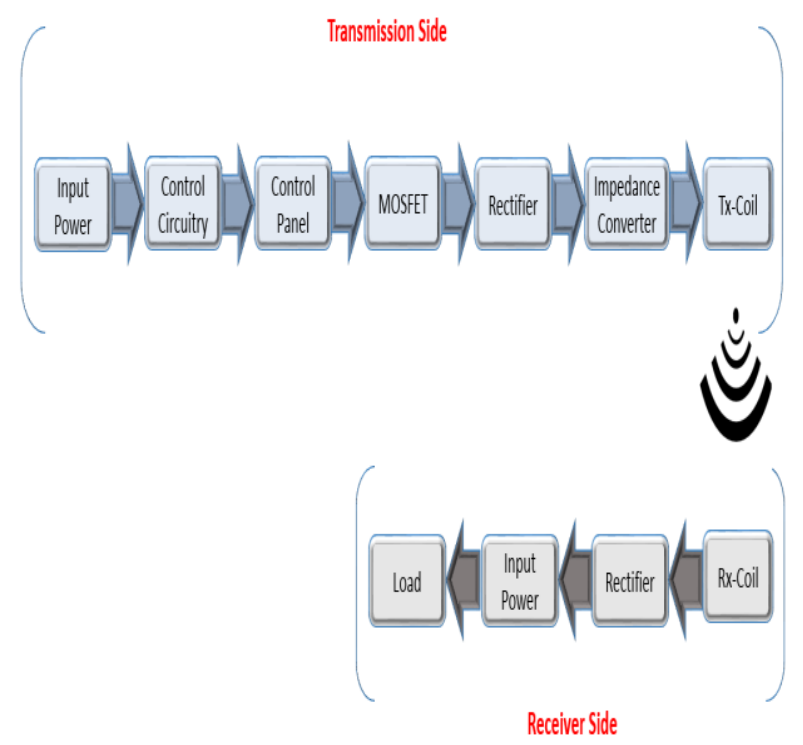

Figure 4. A simple Schematic Representation of Witricity

A more beneficial insight would be that with WiTricity, a number of devices will not require batteries to operate. It can be visualized that this can be applied to our cell phones, household robots, laptop computers, and other portable electronics capable of charging themselves. This will not require them plugging in and get rid of ubiquitous power wire. The need of bulky batteries shall diminish. Given below fig. 5 shows a setup used for wireless transmission of electric energy from source to load.

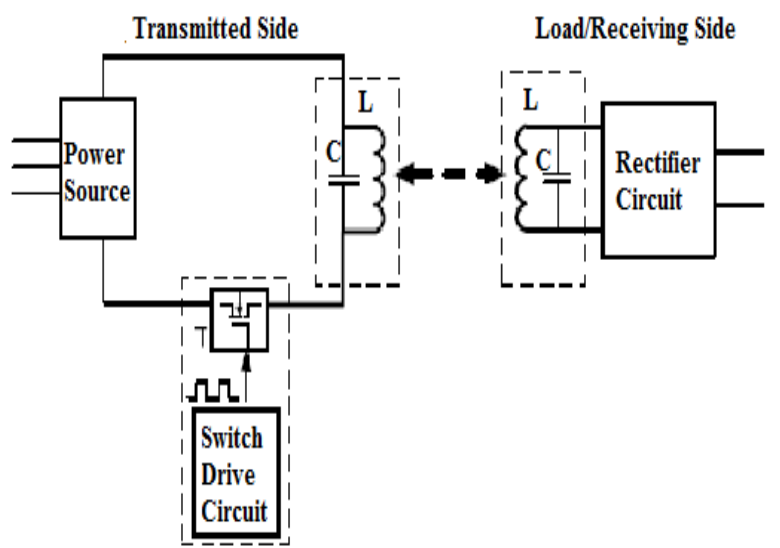

Figure 5. A simple Schematic Representation of Witricity 


\section{Conclusion}

A review of various techniques for wireless transfer is presented. The Wireless Electricity technology relies on the magnetic field in vicinity for power transfer, rendering it a non-radioactive mode of energy transfer. It is a well-documented fact that biological organisms, people, and animals interact very weakly with the magnetic fields and thus this technology can be regarded as safe. The potential for transfer depends upon the source and receivers, irrespective of presence of obstacles between them. With the proximity of both, the efficiency can exceed $95 \%$ which is measured primarily by the distance between the power sources and capture device, however, the shape may impact the efficiency.

Conventional magnetic induction is based upon the near proximity of both power source and receiver device. For efficient transfer, the near proximity could be defined as few millimetres. With the recent advent of driving loop and load loop, the flexibility in transmission distance has been extended. Sharp resonant strong coupling forms the basis of wireless electricity technology. They allow the efficient power transfer even when the distances between the power source and capture device are several times the size of the devices themselves.

\section{Acknowledgment}

The authors would like to convey their gratefulness to Universiti Teknologi PETRONAS, for their assistance, guidance and support in all the phases of this project.

\section{References}

1. A. Karalis, J. D. Joannopoulos, and M. Soljačić, "Efficient wireless non-radiative mid-range energy transfer," Annals of Physics, vol. 323, pp. 34-48, (2008).

2. N. Tesla, "Apparatus for transmitting electrical energy," ed: Google Patents, 1914.

3. R. Lomas, The man who invented the twentieth century: Headline, (1999).

4. E. S. Hochmair, "System optimization for improved accuracy in transcutaneous signal and power transmission," Biomedical Engineering, IEEE Transactions on, pp. 177-186, (1984).

5. A. W. Green and J. Boys, "10 kHz inductively coupled power transfer-concept and control," in Power Electronics and Variable-Speed Drives, 1994. Fifth International Conference on, (1994), pp. 694-699.

6. C. Zhu, K. Liu, C. Yu, M. Rui, and H. Cheng, "Simulation and experimental analysis on wireless energy transfer based on magnetic resonances," in Vehicle Power and Propulsion Conference, 2008. VPPC'08. IEEE, (2008), pp. $1-4$.
7. Y.-H. Kim, S.-Y. Kang, S. Cheon, M.-L. Lee, J.M. Lee, and T. Zyung, "Optimization of wireless power transmission through resonant coupling," in Power Electronics Electrical Drives Automation and Motion (SPEEDAM), (2010) International Symposium on, 2010, pp. 10691073.

8. W. Fu, B. Zhang, D. Qiu, and W. Wang, "Analysis of transmission mechanism and efficiency of resonance coupling wireless energy transfer system," in Electrical Machines and Systems, 2008. ICEMS 2008. International Conference on, (2008), pp. 2163-2168.

9. B. L. Cannon, J. F. Hoburg, D. D. Stancil, and S. C. Goldstein, "Magnetic resonant coupling as a potential means for wireless power transfer to multiple small receivers," Power Electronics, IEEE Transactions on, vol. 24, pp. 1819-1825, (2009).

10. T. C. Beh, T. Imura, M. Kato, and Y. Hori, "Basic study of improving efficiency of wireless power transfer via magnetic resonance coupling based on impedance matching," in Industrial Electronics (ISIE), 2010 IEEE International Symposium on, (2010), pp. 2011-2016.

11. S. Ho, J. Wang, W. Fu, and M. Sun, "A comparative study between novel witricity and traditional inductive magnetic coupling in wireless charging," Magnetics, IEEE Transactions on, vol. 47, pp. 1522-1525, (2011).

12. Y. Burali and C. Patil, "Wireless electricity transmission based on electromagnetic and resonance magnetic coupling," Int. J. of Computational Engr. Res, vol. 3, pp. 48-51, (2012).

13. A. Kurs, A. Karalis, R. Moffatt, J. D. Joannopoulos, P. Fisher, and M. Soljačić, "Wireless power transfer via strongly coupled magnetic resonances," science, vol. 317, pp. 8386, (2007).

14. W. Qiang and L. Hong, "Research on the wireless power transmission system based on coupled magnetic resonances," in Electronics, Communications and Control (ICECC), 2011 International Conference on, (2011), pp. 22552258.

15. A. E. Gundogdu and E. Afacan, "Some experiments related to wireless power transmission," in Cross Strait Quad-Regional Radio Science and Wireless Technology Conference (CSQRWC), 2011, (2011), pp. 507509.

16. P. Jae-Hyun, P. Byung-Chul, L. Jeong-Hae, R. Young-Ho, P. Eun-Seok, and K. Sang-Wook, "Optimum frequency of high Q-factor resonator for magnetic resonance coupling," in Microwave Conference (EuMC), 2011 41st European, 2011, pp. 61-63.

17. Y. Kawamura and M. Shoyama, "Wireless power transmission using LC cancellation," in ECCE Asia Downunder (ECCE Asia), 2013 IEEE, (2013), pp. 1041-1045. 
18. K. Seong-Min, I. K. Cho, J. I. Moon, S. I. Jeon, and J. I. Choi, "5W wireless power transmission system with coupled magnetic resonance," in Microwave, Antenna, Propagation and EMC Technologies for Wireless Communications (MAPE), 2013 IEEE 5th International Symposium on, (2013), pp. 255-258.

19. J. Bing, J. R. Smith, M. Philipose, S. Roy, K. Sundara-Rajan, and A. V. Mamishev, "Energy Scavenging for Inductively Coupled Passive RFID Systems," in Instrumentation and Measurement Technology Conference, 2005. IMTC 2005. Proceedings of the IEEE, (2005), pp. 984-989.

20. K. Tashiro, H. Wakiwaka, S. Inoue, and Y. Uchiyama, "Energy Harvesting of Magnetic Power-Line Noise," Magnetics, IEEE Transactions on, vol. 47, pp. 4441-4444, (2011).

21. M. Takato, T. Nishi, M. Kaneko, J. Tanida, S. Tada, K. Saito, et al., "Multilayer ceramic coil for wireless power transfer system by photo resist film process," in Electronics Packaging (ICEP), 2014 International Conference on, (2014), pp. 326-331.

22. B. H. Soong, Y. L. Sum, W. Liu, and S. Ramachandran, "Characterizing wire wound inductor coils for optimized wireless power transfer," in Advanced Intelligent Mechatronics, 2009. AIM 2009. IEEE/ASME International Conference on, (2009), pp. 469-474.

23. N. S. Artan, R. C. Patel, N. Chengzhi, and H. J. Chao, "High-efficiency wireless power delivery for medical implants using hybrid coils," in Engineering in Medicine and Biology Society (EMBC), (2012) Annual International Conference of the IEEE, 2012, pp. 1683-1686.

24. K. Tashiro, "Broadband Air-Core Brooks-Coil Induction Magnetometer," in SICE-ICASE, 2006. International Joint Conference,

25. (2006), pp. 179-182.

26. N. O. Sokal and A. D. Sokal, "Class E-A new class of high-efficiency tuned single-ended switching power amplifiers," Solid-State Circuits, IEEE Journal of, vol. 10, pp. 168-176, (1975).

27. M. Acar, A. J. Annema, and B. Nauta, "Generalized Design Equations for Class-E Power Amplifiers with Finite DC Feed Inductance," in Microwave Conference, 2006. 36th European, (2006), pp. 1308-1311.

28. R. A. C. Wei-Ting Chen, Shuhei Yoshida, Jenshan Lin, and Chao-Kai Hsu, "A 36W Wireless Power Transfer System with $82 \%$ Efficiency for LED Lighting Applications," The Japan Institute of Electronics Packaging vol. 6, pp. 32-36, (2013).

29. W. Chen, R. A. Chinga, S. Yoshida, J. Lin, C. Chen, and W. Lo, "A 25.6 W $13.56 \mathrm{MHz}$ wireless power transfer system with a $94 \%$ efficiency GaN Class-E power amplifier," in Microwave Symposium Digest (MTT), 2012 IEEE MTT-S International, (2012), pp. 1-3.

30. T. Sekitani, M. Takamiya, Y. Noguchi, S. Nakano, Y. Kato, T. Sakurai, et al., "A largearea wireless power-transmission sheet using printed organic transistors and plastic MEMS switches," Nature materials, vol. 6, pp. 413-417, (2007).

31. T. Imura and Y. Hori, "Wireless power transfer using electromagnetic resonant coupling," Journal of The Institute of Electrical Engineers of Japan, vol. 129, pp. 414-417, (2009).

32. Q. Yuan, Q. Chen, L. Li, and K. Sawaya, "Numerical analysis on transmission efficiency of evanescent resonant coupling wireless power transfer system," Antennas and Propagation, IEEE Transactions on, vol. 58, pp. 1751-1758, (2010).

33. C.-J. Chen, T.-H. Chu, C.-L. Lin, and Z.-C. Jou, "A study of loosely coupled coils for wireless power transfer," Circuits and Systems II: Express Briefs, IEEE Transactions on, vol. 57, pp. 536-540, (2010). 